\title{
ON THE ARITHMETIC OF ORDER TYPES
}

BY

ANNE C. MOREL

1. Introduction. This paper deals with the arithmetic of order types, in particular, with finite multiplication $\left({ }^{1}\right)$. In the first part, the question of what order types $\alpha$ are left cancelling types is investigated, i.e., what are the types $\alpha$ such that $\alpha \cdot \beta=\alpha \cdot \gamma$ implies $\beta=\gamma$ for all types $\beta$ and $\gamma$. A necessary and sufficient condition for a type $\alpha$ to be a left cancelling type is obtained. Several particular results are found, among them that every scattered type is a left cancelling type. In the second part we investigate solutions of the equation $\xi^{n}=\alpha$. Some sufficient conditions for the equation to have at most one solution are found. Moreover, it turns out that one can find denumerable types $\alpha$ for which the equation $\xi^{n}=\alpha$ has exactly $\mathfrak{m}$ solutions, where $\mathfrak{m}$ is any finite cardinal, or $\mathfrak{m}=\boldsymbol{\aleph}_{0}$ or $\mathfrak{m}=2 \boldsymbol{\aleph}_{0}$.

2. Some definitions and elementary consequences $\left({ }^{2}\right)$. The term relation will be used to denote any binary relation. The field of the relation $R$, in symbols, $F(R)$, is the set consisting of all first elements $x$ and all second elements $y$ of the ordered pairs $\langle x, y\rangle$ belonging to $R$. If $R \supseteq R^{\prime}$, then $R^{\prime}$ is said to be a subrelation of $R$.

All relations considered (but for some obvious exceptions) are assumed to be (simply) ordering relations, i.e., they are anti-symmetric, connected and transitive.

The relation $R$ is isomorphic to the relation $S$, in symbols, $R \cong S$, if there exists a one-to-one function $f$ which maps $F(R)$ onto $F(S)$ and fulfills the following condition: for any $x, y \in F(R)$, if $x R p$ then $f(x) S f(y)$. (Hence, since $R$ and $S$ are simply ordered, $f(x) S f(y)$ implies $x R y$.) If $f$ is such a function, we write $R \cong_{f} S$.

The symbol $\tau(R)$ will be used to denote the order type of the relation $R$. In general, Greek lower case letters $\alpha, \beta, \gamma, \cdots$ will be used to designate order types, although finite order types (which can of course, be considered as natural numbers) will also be denoted by $m, n, p, \cdots$.

Received by the editors February 7, 1955 and, in revised form, December 15, 1957.

(1) The results presented in this article were obtained in 1951-1954 and stated without proof in several abstracts and notes; see the Bibliography at the end of the paper. A large part of the results was included in the author's doctoral dissertation, accepted by the University of California in January, 1953. A portion of the preparation of the paper was done while the author was working on a research project in the foundation of mathematics sponsored by the Office of Ordnance Research, U. S. Army, and directed by Professor Alfred Tarski. The author wishes to express thanks to Professor Tarski, under whose direction the dissertation was written.

(2) For many of the notions introduced in this section, and their elementary properties, see Hausdorff [10] or Sierpinski [12]. 
We use the symbol $\kappa(A)$ for the power of the set $A$. The word denumerable will here mean either finite or of power $\boldsymbol{\aleph}_{0}$. A relation type $\alpha$ will be said to be finite or infinite, according as $F(R)$ is finite or infinite, where $R$ is any relation such that $\tau(R)=\alpha$. For any sets $A$ and $B$, we write $A \times B$ for the Cartesian product of $A$ and $B$.

It is assumed that the reader is familiar with the definitions of the ordinal sum, $R+S$, and the ordinal product, $R \cdot S$, of the relations $R$ and $S$. Suppose that $R$ is a relation and that, for every $x \in F(R), G(x)$ is a relation, and moreover, $G(x) \cap G\left(x^{\prime}\right)=\varnothing$ for distinct elements $x, x^{\prime} \in F(R)$. Then the ordered sum of the relations $G(x)$ over $R, \sum_{R} G(x)$, is defined as follows:

$$
\sum_{R} G(x)=\bigcup_{x \in F(R)} G(x) \cup \bigcup_{x R x^{\prime}, x \neq x^{\prime}}\left[F(G(x)) \times F\left(G\left(x^{\prime}\right)\right)\right] .
$$

It is easily seen that $\sum_{R} G(x)$ is again an ordering relation. The more familiar operations of ordinal addition and ordinal multiplication can be viewed as special cases of the general operation defined above. First, consider the case where $F(R)=\{y, z\}$, with $y \neq z$ and $y R z$. Then $\sum_{R} G(x)$ reduces to the ordinal sum, $G(y)+G(z)$. Secondly, suppose that all the $G(x)$ are isomorphic to some relation $S$. Then $\sum_{R} G(x) \cong S \cdot R$. Now for each $x \in F(R)$, let $\gamma(x)$ be the order type of $G(x)$. Then the ordinal sum of the types $\gamma(x)$ over $R, \sum_{R} \gamma(x)$, is defined as follows:

$$
\sum_{R} \gamma(x)=\tau\left(\sum_{R} G(x)\right)
$$

The symbols $\alpha+\beta$ and $\alpha \cdot \beta$ stand for the ordinal sum and ordinal product respectively of the types $\alpha$ and $\beta$.

For every type $\alpha$ and for every natural number $n$ we put

$$
\alpha^{0}=1, \alpha^{n+1}=\alpha^{n} \cdot \alpha .
$$

The type of the empty relation will be denoted by $0 ; \omega$ and $\omega^{*}$ are the types of the non-negative and negative integers respectively (with their usual ordering), $\eta$ the type of the rationals and $\lambda$ the type of the reals.

For any relation $R$ and any set $N$, we put $R\langle N\rangle=R \cap(N \times N)$. The set $M$ will be called an interval of the relation $R$ if $M \subseteq F(R)$ and if, whenever $y, z \in M, x \in F(R), y R x$ and $x R z$, then $x \in M$. We use the symbols $(x, y)_{R}$ and $[x, y]_{R}$ respectively for the open and closed intervals of $R$ with endpoints $x$ and $y$. It is convenient to assume here that $(x, y)_{R}=(y, x)_{R}$ and that $[x, y]_{R}$ $=[y, x]_{R}$. The subscript will be omitted whenever no ambiguity results. A relation $R$ (or type $\tau(R)$ ) is dense if $\kappa(F(R))>1$ and if, for every pair of distinct elements $x, y \in F(R), \kappa([x, y])>2$. A relation $S$ (or type $\tau(S)$ ) is scattered if $\kappa(F(S)) \geqq 1$ and if $S$ has no dense subrelation. We call a relation $T$ (or type $\tau(T))$ elementary if $T \neq \varnothing$ and if, for every $x, y \in F(T), \kappa([x, y])$ is finite. We shall designate the class of elementary types by $\mathbf{E}$; it is almost evident that $\mathrm{E}$ consists of all nonzero finite types and of the types $\omega, \omega^{*}$ and $\omega^{*}+\omega$. 
The following lemma is very simple and we shall make use of it hereafter without giving an explicit reference.

Lemma. Suppose that $\alpha, \beta$ and $\gamma$ are order types, that $R$ is an ordering relation and that, for each $x \in F(R), \gamma(x)$ is an order type. Then

(i) $\alpha+(\beta+\gamma)=(\alpha+\beta)+\gamma$,

(ii) $\alpha \cdot(\beta \cdot \gamma)=(\alpha \cdot \beta) \cdot \gamma$,

(iii) $\alpha \cdot \sum_{R} \gamma(x)=\sum_{R}(\alpha \cdot \gamma(x))$.

3 . Cancellation theorems for products of order types. The following decomposition theorem, 3.1, was obtained jointly by Tarski and the author.

THEOREM 3.1. Let $\mathrm{K}$ be any class of order types satisfying the condition

(a) For any types $\alpha$ and $\beta, \alpha+1+\beta \in \mathrm{K}$ if and only if $\alpha, \beta \in \mathrm{K}$.

Let $\Lambda$ be the class of all nonzero types $\alpha$ such that

(b) Whenever $\alpha$ is expressible in the form $\alpha=\beta+1+\gamma+1+\delta$, then $\gamma \in \mathrm{K}$. Then

(i) For every type $\alpha \neq 0$, there exists a relation $R$ and a function $\gamma \cdot$ on $F(R)$ to $\Lambda$ such that $\alpha=\sum_{R} \gamma(x)$, while if $x_{1}, x_{2} \in F(R)$ and $x_{1} \neq x_{2}$, then

$$
\sum_{R\left\langle\left[x_{1}, x_{2}\right]\right\rangle} \gamma(x) \notin \Lambda \text {. }
$$

(ii) If $\sum_{R} \gamma(x)$ and $\sum_{S} \delta(y)$ are any two representations of $\alpha$ of the form described in (i), then there exists a function $f$ such that $R \cong_{f} S$ and $\gamma(x)=\delta(f(x))$ for each $x \in F(R)$.

Proof. Let $T$ be a relation of type $\alpha$, where $\alpha \neq 0$. Define the relation $V$ on $F(T)$ as follows:

$$
t_{1} V t_{2} \text { if and only if } t_{1}=t_{2} \text { or } \tau\left(T\left\langle\left(t_{1}, t_{2}\right)\right\rangle\right) \in \mathrm{K} .
$$

Clearly $V$ is reflexive and symmetric; that $V$ is transitive follows from (a), and hence $V$ is an equivalence relation with $F(V)=F(T)$.

Using (a) again, we obtain:

If $X_{1}, X_{2}$ are distinct equivalence classes defined by $V$, and if $t_{1} \in X_{1}$ (2) $t_{2} \in X_{2}$ and $t_{1} T t_{2}$, then, for every $t^{(1)} \in X_{1}$ and every $t^{(2)} \in X_{2}$, we have $t^{(1)} T t^{(2)}$.

Let $X$ be any equivalence class and put $\zeta=\tau(T\langle X\rangle)$. Obviously $\zeta \neq 0$. Suppose $\zeta=\beta+1+\gamma+1+\delta$ for some $\beta, \gamma$ and $\delta$. Then there must exist subrelations $T_{1}, T_{2}$ and $T_{3}$ of $T\langle X\rangle$ and elements $t_{1}, t_{2} \in X$ such that

$$
T\langle X\rangle=T_{1}+\left\{\left\langle t_{1}, t_{1}\right\rangle\right\}+T_{2}+\left\{\left\langle t_{2}, t_{2}\right\rangle\right\}+T_{3} .
$$

Since, by (1), $\tau\left(T\left\langle\left(t_{1}, t_{2}\right)\right\rangle\right)=\tau\left(T_{2}\right) \in \mathbf{K}$, it follows from (b) that $\zeta \in \Lambda$.

Now let $R$ be the relation between equivalence classes such that $X_{1} R X_{2}$ 
if and only if either $X_{1}=X_{2}$ or there exists elements $t_{1} \in X_{1}$ and $t_{2} \in X_{2}$ such that $t_{1} T t_{2}$.

From (2), it follows that $R$ is an ordering relation; moreover we have

$$
T=\sum_{R} T\langle X\rangle .
$$

Finally, assume that $\tau\left(\sum_{R\left\langle\left[X_{1}, X_{2}\right]\right\rangle} T\langle X\rangle\right) \in \Lambda$, with $X_{1} \neq X_{2}$. Choose $t_{1} \in X_{1}$ and $t_{2} \in X_{2}$. By (b), $\tau\left(T\left\langle\left(t_{1}, t_{2}\right)\right\rangle\right) \in \mathrm{K}$, and hence by (1) we have $t_{1} V t_{2}$, which is a contradiction. Thus (i) has been established.

Now suppose that $\sum_{R} \gamma(x)$ and $\sum_{S} \delta(y)$ are two representations of $\alpha$ satisfying the hypothesis of (ii). Choose relations $G(x)$ and relations $H(y)$ slich that

(3) $\tau(G(x))=\gamma(x)$ for every $x \in F(R), \tau(H(y))=\delta(y)$ for every $y \in F(S)$, and

$$
\sum_{R} G(x)=\sum_{S} H(y) .
$$

We shall establish the following fact:

(5) For each $x \in F(R)$ there exists a $y \in F(S)$ such that $F(G(x))=F(H(y))$.

Let $x$ be any element of $F(R)$; pick an element $u \in F(G(x))$. Then, by (4), there exists a unique $y \in F(S)$ such that $u \in F(H(y))$. Let $u^{\prime}$ be any element such that

$$
u^{\prime} \neq u \text { and } u^{\prime} \in F(G(x)) .
$$

Our next step is to prove, by contradiction, that

$$
u^{\prime} \in F(H(y)) \text {. }
$$

Assume for the moment that (6) fails. Let us suppose, for definiteness, that $u G(x) u^{\prime}$ (rather than $\left.u^{\prime} G(x) u\right)$. Then there is exactly one $y^{\prime} \in F(S)$ for which

$$
u^{\prime} \in F\left(H\left(y^{\prime}\right)\right), y S y^{\prime} \text { and } y \neq y^{\prime} \text {. }
$$

We put

$$
W=\sum_{S\left(\left\{y, y^{\prime}\right]\right\}} H(y) \text { and } \beta=\tau(W) .
$$

Using the hypotheses, (3) and (7), we find that

$$
\beta \notin \Lambda \text {. }
$$

Consider a decomposition of $W$ of the following sort:

$$
W=W_{1}+\left\{\left\langle v_{1}, v_{1}\right\rangle\right\}+W_{2}+\left\{\left\langle v_{2}, v_{2}\right\rangle\right\}+W_{3} .
$$

Our argument now divides into cases, depending upon the relative order in 
$W$ of the elements $u, u^{\prime}, v_{1}$ and $v_{2}$. We treat only the case where $u, u^{\prime}, v_{1}$ and $v_{2}$ are distinct elements such that

$$
v_{1} W u, u W v_{2} \text { and } v_{2} W u^{\prime}
$$

the other cases are quite analogous. We note, using (7) and (10), that $v_{1} \in F(H(y))$. Since $u, v_{1} \in F(H(y))$, we infer from (b), the hypotheses and (3) that

$$
\tau\left(W\left\langle\left(v_{1}, u\right)\right\rangle\right) \in \mathrm{K} .
$$

Similarly, since $u, u^{\prime} \in F(G(x))$, we have

$$
\tau\left(W\left\langle\left(u, u^{\prime}\right)\right\rangle\right) \in \mathrm{K} .
$$

A consequence of (a), (10), (11) and (12) is

$$
\tau\left(W\left\langle\left(v_{1}, u^{\prime}\right)\right\rangle\right) \in \mathrm{K}
$$

from (a), (10), and (13), we conclude that

$$
\tau\left(W\left\langle\left(v_{1}, v_{2}\right)\right\rangle\right) \in \mathrm{K} .
$$

Now by using (b), (7), (9) and (14), we obtain $\beta \in \Lambda$, in contradiction to (8); thus (6) has been verified.

An immediate consequence of (6) is

$$
F(G(x)) \subseteq F(H(y))
$$

by a symmetric argument we could as well have obtained

$$
F(H(y)) \subseteq F(G(x)) .
$$

Hence (5) has finally been proved. Moreover, (3) and (5) imply that

$$
\gamma(x)=\delta(y) \text {. }
$$

We are now able to define the function $f$ of (ii) as follows: for each $x \in F(R)$, let $f(x)$ be the unique $y \in F(S)$ such that $F(G(x))=F(H(y))$. The proof that $f$ satisfies the requisite conditions is almost immediate.

We recall that $\mathbf{E}$ denotes the class of all elementary types.

COROLLARY 3.2. For every type $\alpha \neq 0$ there exists a relation $R$ and a function $\gamma$ on $F(R)$ to $\mathrm{E}$ such that $\alpha=\sum_{R} \gamma(x)$, while if $x$ immediately precedes $x^{\prime}$ with respect to $R$, then $g(x)+g\left(x^{\prime}\right) \notin \mathrm{E}$. This representation is essentially unique (in the sense of 3.1 (ii)).

Proof. Let $\mathrm{K}$ be the class of finite types (including 0 ). Clearly $\mathrm{K}$ satisfies hypothesis (a) of 3.1. The set $\Lambda$ of 3.1 then consists of all elementary types.

A representation of a type $\alpha$ as described above will be called an elementary decomposition of $\alpha$. We shall also use the notion (defined in the obvious way) of an elementary decomposition of a relation. 
COROLLARY 3.3. For every type $\alpha \neq 0$ exactly one of the following holds:

(i) $\alpha$ is a scattered type.

(ii) $\alpha$ is (essentially uniquely) representable in the form $\alpha=\sum_{R} \gamma(x)$, where $R$ is a dense relation and, for each $x \in F(R), \gamma(x)$ is scattered $\left({ }^{3}\right)$.

Proof. For $\mathrm{K}$ of 3.1 take the class consisting of all scattered types and 0 . Then $\Lambda$ is precisely the class of scattered types, and by 3.1 we obtain $\alpha=\sum_{R} \gamma(x)$, where each $\gamma(x)$ is scattered but $\sum_{R\left\langle\left[x_{1}, x_{2}\right]\right\rangle} \gamma(x)$ is not scattered whenever $x_{1} \neq x_{2}$. If $\tau(R)=1$, we obtain (i). Consider the case $\tau(R) \neq 1$. If $R$ is not dense, there must exist two consecutive elements, $x^{\prime}, x^{\prime \prime} \in F(R)$. Hence $\sum_{R\left\langle\left[x^{\prime}, x^{\prime \prime}\right]\right\rangle} \gamma(x)=\gamma\left(x^{\prime}\right)+\gamma\left(x^{\prime \prime}\right)$ is not scattered, but, as is well known, the sum of two scattered types is again scattered. Hence $R$ is dense.

The representation of $\alpha$ described above will be called a scattered decomposition of $\alpha$.

We state without proof the following two elementary lemmas.

LEMMA 3.4. If $\alpha+\beta=\gamma+\delta$, then either

(i) There is a type $\epsilon$ such that $\alpha=\gamma+\epsilon$ and $\delta=\epsilon+\beta$,

or

(ii) There is a type $\epsilon$ such that $\gamma=\alpha+\epsilon$ and $\beta=\epsilon+\delta$.

Lemma 3.5. If $\alpha \cdot \beta=\gamma+\delta$, then at least one of the following must hold:

(i) There exist types $\beta_{1}$ and $\beta_{2}$ such that $\gamma=\alpha \cdot \beta_{1}, \delta=\alpha \cdot \beta_{2}$ and $\beta_{1}+\beta_{2}=\beta$,

(ii) There exist nonzero types $\alpha_{1}$ and $\alpha_{2}$ with $\alpha_{1}+\alpha_{2}=\alpha$, and types $\beta_{1}$ and $\beta_{2}$ with $\beta_{1}+1+\beta_{2}=\beta$, such that $\gamma=\alpha \cdot \beta_{1}+\alpha_{1}$ and $\delta=\alpha_{2}+\alpha \cdot \beta_{2}$.

If $\alpha, \beta, \gamma$ and $\delta$ are any types such that $\alpha=\beta+\gamma+\delta$, then $\beta, \gamma$ and $\delta$ are known respectively as initial, middle and final portions of $\alpha$; in symbols, $\beta \mathfrak{g} \alpha, \gamma \mathfrak{T} \alpha$ and $\delta \mathfrak{F} \alpha$. For the negations of the three preceding expressions we write $\beta \overline{\mathscr{g}} \alpha, \gamma \overline{\mathfrak{T}} \alpha$ and $\delta \overline{\mathscr{F}} \alpha$.

THEOREM 3.6. If $\alpha \cdot \beta=\gamma \cdot \delta$, then at least one of the following conditions is satisfied:

(i) $\beta=\delta$,

(ii) There exist types $\gamma_{1}, \gamma_{2}$ and an infinite type $\delta^{\prime}$ such that $\alpha=\gamma_{1}+\gamma \cdot \delta^{\prime}+\gamma_{2}$, $\gamma_{1} \mathcal{F} \gamma, \gamma_{2} \mathfrak{g} \gamma$ and $\delta^{\prime} \mathfrak{T l} \delta$,

(3) Corollary 3.3 can be found in Hausdorff [10, p. 95]; however, it is not stated there that the representation is unique. By following the lines of Hausdorff's argument, the author first obtained Corollary 3.2, and then the more general Theorem 3.1 was established. In addition to 3.2 and 3.3, some other rather particular cases of 3.1 can be obtained. For instance, as is easily seen, the premises of 3.1 are satisfied if we take for $K$ the class of all types of dense relations without endpoints and for $\Lambda$ the class consisting of 1 and of all dense types. Corollary 3.2 can be generalized by taking for $\mathrm{K}$ the class of types of power less than $\aleph_{\mu}, \mu$ an arbitrary ordinal, and for $\Lambda$ the class of all types $\alpha$ such that every closed interval of $\alpha$ is of power less than $\aleph_{\mu}$. As can be shown, $\Lambda$ consists of all types $\alpha$ representable in the form $\alpha=\sum_{R} \phi(x)$, where each $\phi(x)$ is of power less than $\aleph_{\mu}$ and $\tau(R)=\omega_{\mu}^{*}+\omega_{\mu}$. 
(iii) There exist types $\alpha_{1}, \alpha_{2}$ and an infinite type $\beta^{\prime}$ such that $\gamma=\alpha_{1}+\alpha \cdot \beta^{\prime}$ $+\alpha_{2}, \alpha_{1} \mathcal{F} \alpha, \alpha_{2} \mathfrak{g} \alpha$ and $\beta^{\prime} \mathfrak{M} \beta$,

(iv) $\alpha \cdot n=\gamma \cdot p$, where $n$ and $p$ are distinct finite types.

Proof. If $\alpha \cdot \beta=0$, then either (i) or (iv) holds. We assume now that $\alpha \cdot \beta \neq 0$. Choose relations $R_{\alpha}, R_{\beta}, S_{\gamma}$ and $S_{\delta}$ such that

$$
\tau\left(R_{\alpha}\right)=\alpha, \tau\left(R_{\beta}\right)=\beta, \tau\left(S_{\gamma}\right)=\gamma \text { and } \tau\left(S_{\delta}\right)=\delta .
$$

From 3.2 we obtain

(2) $R_{\beta}=\sum_{R} R^{\prime}(x)$, where $\sum_{R} R^{\prime}(x)$ is an elementary decomposition of $R_{\beta}$,

(3) $S_{\delta}=\sum_{S} S^{\prime}(y)$, where $\sum_{S} S^{\prime}(y)$ is an elementary decomposition of $S_{\delta}$.

Let

$$
T=R_{\alpha} \cdot \sum_{R} R^{\prime}(x), \quad U=S_{\gamma} \cdot \sum_{S} S^{\prime}(y) .
$$

Then, for some function $f$,

$$
T=\sum_{R}\left(R_{\alpha} \cdot R^{\prime}(x)\right) \cong_{f} \sum_{S}\left(S_{\gamma} \cdot S^{\prime}(y)\right)=U .
$$

From (4) (using the symbol $f^{+}(A)$ to denote the set of all $f(x)$ with $x \in A$ ) we readily obtain three cases:

CASE I. For every $x \in F(R)$ there is a $y \in F(S)$ such that

$$
f^{+}\left(F\left(R_{\alpha} \cdot R^{\prime}(x)\right)=F\left(S_{\gamma} \cdot S^{\prime}(y)\right) .\right.
$$

CASE II. There exist a $y \in F(S)$ and distinct elements $x_{1}, x_{2} \in F(R)$ such that

$$
\begin{aligned}
& f^{+}\left(F\left(R_{\alpha} \cdot R^{\prime}\left(x_{1}\right)\right)\right) \cap F\left(S_{\gamma} \cdot S^{\prime}(y)\right) \neq \varnothing, \\
& f^{+}\left(F\left(R_{\alpha} \cdot R^{\prime}\left(x_{2}\right)\right)\right) \cap F\left(S_{\gamma} \cdot S^{\prime}(y)\right) \neq \varnothing .
\end{aligned}
$$

CASE III. There exist an $x \in F(R)$ and distinct elements $y_{1}, y_{2} \in F(S)$ such that

$$
\begin{aligned}
& f^{+}\left(F\left(R_{\alpha} \cdot R^{\prime}(x)\right)\right) \cap F\left(R_{\gamma} \cdot R^{\prime}\left(y_{1}\right)\right) \neq \varnothing, \\
& f^{+}\left(F\left(R_{\alpha} \cdot R^{\prime}(x)\right)\right) \cap F\left(R_{\gamma} \cdot R^{\prime}\left(y_{2}\right)\right) \neq \varnothing .
\end{aligned}
$$

Suppose that Case I holds. Let $g$ be the function on $F(R)$ such that, for each $x \in F(R), g(x)$ is the unique $y$ for which $f^{+}\left(F\left(R_{\alpha} \cdot R^{\prime}(x)\right)\right)=F\left(S_{\gamma} \cdot S^{\prime}(y)\right)$. By (4) we have $R \cong_{0} S$. If, moreover, for each $x \in F(R), \tau\left(R^{\prime}(x)\right)=\tau\left(S^{\prime}(g(x))\right)$, then by (1), (2) and (3) we obtain conclusion (i) of our lemma. Suppose, on the other hand, that there does exist an $x \in F(R)$ such that $\tau\left(R^{\prime}(x)\right)$ $\neq \tau\left(S^{\prime}(g(x))\right)$. Putting $\phi(x)=\tau\left(R^{\prime}(x)\right)$ and $\theta(x)=\tau\left(S^{\prime}(g(x))\right)$, we have, by (1), (2) and (3), $\alpha \cdot \phi(x)=\gamma \cdot \theta(x)$, where $\phi(x) \neq \theta(x)$ and $\phi(x), \theta(x) \in \mathrm{E}$. If $\phi(x)$ and $\theta(x)$ are both finite types, we get conclusion (iv). If not, we have

$\alpha \cdot \phi(x)=\gamma \cdot \theta(y)$, with $\phi(x) \neq \theta(y), \phi(x), \theta(y) \in \mathrm{E}, \phi(x) \mathfrak{T} \beta, \theta(y)$ MT $\delta$ and $\phi(x)+\theta(y)$ infinite. 
It will be shown at the conclusion of the proof that (5) implies either (ii) or (iii).

Now assume that Case II holds. We may then choose elements $\left\langle t_{1}, u_{1}\right\rangle$ $\in F\left(R_{\alpha} \cdot R^{\prime}\left(x_{1}\right)\right),\left\langle t_{2}, u_{2}\right\rangle \in F\left(R_{\alpha} \cdot R^{\prime}\left(x_{2}\right)\right)$, and $\left\langle v_{1}, w_{1}\right\rangle,\left\langle v_{2}, w_{2}\right\rangle \in F\left(S_{\gamma} \cdot S^{\prime}(y)\right)$ such that $f\left(\left\langle t_{1}, u_{1}\right\rangle\right)=\left\langle v_{1}, w_{1}\right\rangle$ and $f\left(\left\langle t_{2}, u_{2}\right\rangle\right)=\left\langle v_{2}, w_{2}\right\rangle$. Then, by (2) and (3), $\left[u_{1}, u_{2}\right]_{R_{\beta}}$ is infinite while $\left[w_{1}, w_{2}\right]_{S_{\delta}}$ is finite. Let

$$
N=F\left(S_{\gamma}\right) \times\left[w_{1}, w_{2}\right] .
$$

We see from (1), (3) and (4) that $N$ is an interval of $U$ and that

$$
\tau(U\langle N\rangle)=\gamma \cdot n \text {, where } n \text { is a finite type and } n \neq 0 .
$$

Put

$$
M=\left(f^{-1}\right)+(N) .
$$

Obviously, $M$ is an interval of $T$. We denote by $Q$ the set of all second terms of the ordered pairs of $M$.

If each $u \in Q$ satisfies the condition $F\left(R_{\alpha}\right) \times\{u\} \subseteq M$, then, clearly, $M=F\left(R_{\alpha}\right) \times Q$; using (1), (2) and the fact that $u_{1}, u_{2} \in Q$, we easily obtain that $\tau(T\langle M\rangle)=\alpha \cdot \beta^{\prime}$, where $\beta^{\prime}$ is infinite and $\beta^{\prime} \mathfrak{T} \beta$. Hence, from (4), (6) and $(7)$ we have

$$
\gamma \cdot n=\alpha \cdot \beta^{\prime} \text {, where } n \text { is finite, } \beta^{\prime} \mathfrak{T} \beta \text { and } \beta^{\prime} \text { is infinite. }
$$

Suppose now that there exists a $u^{(1)} \in Q$ such that $F\left(R_{\alpha}\right) \times\left\{u^{(1)}\right\} \nsubseteq M$. In this case we must have either $u^{(1)}=\min Q$ or $u^{(1)}=\max Q$ (i.e., $u^{(1)} R_{\beta} u$ for every $u \in Q$, or $u R_{\beta} u^{(1)}$ for every $u \in Q$ ). Consider the situation where there is just one such $u^{(1)}$ and $u^{(1)}=\min Q$. Here we may easily verify that $M$ is of the form $M=P \times\left\{u^{(1)}\right\} \cup\left(F\left(R_{\alpha}\right) \times\left(Q-\left\{u^{(1)}\right\}\right)\right)$, where $P \subseteq F\left(R_{\alpha}\right)$ and $R_{\alpha}\langle P\rangle \mathcal{F} R_{\alpha}$. Putting $\alpha_{1}=\tau\left(R_{\alpha}\langle P\rangle\right)$ and $\beta^{\prime}=\tau\left(R_{\beta}\left\langle Q-\left\{u^{(1)}\right\}\right\rangle\right)$, and using (1), (4), (6) and (7), we get

$$
\gamma \cdot n=\alpha_{1}+\alpha \cdot \beta^{\prime}, \text { where } \alpha_{1} \mathscr{F} \alpha, \beta^{\prime} \mathfrak{T H} \beta \text { and } \beta^{\prime} \text { is infinite. }
$$

If there exist distinct types $u^{(1)}, u^{(2)} \in Q$ such that $F\left(R_{\alpha}\right) \times\left\{u^{(1)}\right\} \nsubseteq M$ and $F\left(R_{\alpha}\right) \times\left\{u^{(2)}\right\} \nsubseteq M$, then, by reasoning as above, we obtain the existence of types $\alpha_{1}, \alpha_{2}$ and $\beta^{\prime}$ such that

$$
\boldsymbol{\gamma} \cdot \boldsymbol{n}=\alpha_{1}+\alpha \cdot \beta^{\prime}+\alpha_{2} \text {, where } \alpha_{1} \mathfrak{F} \alpha, \alpha_{2} \mathfrak{g} \alpha, \beta^{\prime} \mathfrak{T} \beta \text { and } \beta^{\prime} \text { is infinite. }
$$

Thus, in order to establish our lemma for Case II, it is sufficient to prove the following statement:

If $\gamma \cdot n=\alpha_{1}+\alpha \cdot \beta^{\prime}+\alpha_{2}$, where $n$ is a nonzero finite type, $\alpha_{1}$ and $\alpha_{2}$ are (8) types such that $\alpha_{1} \mathcal{F} \alpha$ and $\alpha_{2} \mathfrak{g} \alpha$, and $\beta^{\prime}$ is an infinite type with $\beta^{\prime} \mathfrak{T} \beta$, then (iii) holds. 
Obviously (8) is valid for $n=1$; assume that (8) holds for some $n$ and that $\gamma \cdot(n+1)=\alpha_{1}+\alpha \cdot \beta^{\prime}+\alpha_{2}$, where $\alpha_{1} \mathfrak{F} \alpha, \alpha_{2} \mathfrak{g} \alpha, \beta^{\prime} \mathfrak{T} \beta$ and $\beta^{\prime}$ is infinite.

Applying 3.4 to

$$
\gamma \cdot n+\gamma=\left(\alpha_{1}+\alpha \cdot \beta^{\prime}\right)+\alpha_{2}
$$

we obtain either

(9) There is a type $\delta$ such that $\gamma \cdot n=\alpha_{1}+\alpha \cdot \beta^{\prime}+\delta$ and $\alpha_{2}=\delta+\gamma$,

or

(10) There is a type $\delta$ such that $\alpha_{1}+\alpha \cdot \beta^{\prime}=\gamma \cdot n+\delta$ and $\gamma=\delta+\alpha_{2}$.

If (9) holds, then $\delta \mathfrak{g} \alpha_{2}$ and $\alpha_{2} \mathfrak{g} \alpha$; consequently $\delta \mathfrak{g} \alpha$ and by the inductive premise we obtain (iii). If (10) holds, we apply 3.4 again to the first equality of (10) and obtain either

There is a type $\epsilon$ such that $\alpha_{1}=\gamma \cdot n+\epsilon$ and $\delta=\epsilon+\alpha \cdot \beta^{\prime}$,

or

There is a type $\epsilon$ such that $\gamma \cdot n=\alpha_{1}+\epsilon$ and $\alpha \cdot \beta^{\prime}=\epsilon+\delta$.

By (10) and (11), $\gamma=\epsilon+\alpha \cdot \beta^{\prime}+\alpha_{2}$, where $\epsilon \mathfrak{F} \alpha_{1}$, and, by hypothesis, $\alpha_{1} \mathcal{F} \alpha$. Applying 3.5 to the second equality of (12), we have either

(13) There exist types $\beta^{\prime \prime}$ and $\beta^{\prime \prime \prime}$ with $\beta^{\prime \prime}+\beta^{\prime \prime \prime}=\beta^{\prime}, \epsilon=\alpha \cdot \beta^{\prime \prime}$ and $\delta=\alpha \cdot \beta^{\prime \prime \prime}$,

or

(14) There exist types $\beta^{\prime \prime}$ and $\beta^{\prime \prime \prime}$ with $\beta^{\prime \prime}+1+\beta^{\prime \prime \prime}=\beta^{\prime}$ and also types $\alpha_{1}^{\prime}$ and $\alpha_{2}^{\prime}$ with $\alpha_{2}^{\prime}+\alpha_{1}^{\prime}=\alpha$ such that $\epsilon=\alpha \cdot \beta^{\prime \prime}+\alpha_{2}^{\prime}$ and $\delta=\alpha_{1}^{\prime}+\alpha \cdot \beta^{\prime \prime \prime}$.

At least one of the types $\beta^{\prime \prime}$ and $\beta^{\prime \prime \prime}$ of (13) must be infinite; the same is true for (14). Suppose that the type $\beta^{\prime \prime}$ of (13) is infinite. Then, by (12) and (13), we have $\gamma \cdot n=\alpha_{1}+\alpha \cdot \beta^{\prime \prime}$, and our inductive hypothesis is satisfied. If $\beta^{\prime \prime \prime}$ is infinite in (13), then, using (10) we obtain $\gamma=\alpha \cdot \beta^{\prime \prime \prime}+\alpha_{2}$. Similarly, if $\beta^{\prime \prime}$ is infinite in (14), or if $\beta^{\prime \prime \prime}$ is infinite in (14), it follows from (12) or (10) that $\gamma \cdot n=\alpha_{1}+\alpha \cdot \beta^{\prime \prime}+\alpha_{2}^{\prime}$ or $\gamma=\alpha_{1}^{\prime}+\alpha \cdot \beta^{\prime \prime \prime}+\alpha_{2}$. Thus (8) has been proved, and therefore Case II implies (iii). In like manner, (ii) is derivable from Case III.

It remains to be shown that (5) implies either (ii) or (iii). If in (5) either $\phi(x)$ or $\theta(y)$ is finite, then by (8) (or a theorem symmetric to (8)) we obtain either (iii) or (ii). Suppose that $\phi(x)=\omega$ and $\theta(y)=\omega^{*}$. Then

$$
\alpha \cdot \omega=\alpha+\alpha \cdot \omega=\gamma \cdot \omega^{*} .
$$

Applying 3.5 to the second equality of (15), we see that either 
(16) There are nonzero types $\rho_{1}$ and $\rho_{2}$ such that $\alpha=\gamma \cdot \rho_{1}$ and $\rho_{1}+\rho_{2}=\omega^{*}$, or

(17) There are types $\rho_{1}, \rho_{2}$ and $\gamma^{(1)}$ such that $\alpha=\gamma \cdot \rho_{1}+\gamma^{(1)}, \gamma^{(1)} g \boldsymbol{\gamma}$ and $\rho_{1}+1+\rho_{2}=\omega^{*}$.

It follows from (16) that $\rho_{1}=\omega^{*}$; from (5) and the first equation of (16) we obtain (ii). By (17), $\rho_{1} \neq 0$ and hence $\rho_{1}=\omega^{*}$; now (ii) is a consequence of (17).

Assume that $\phi(x)=\omega^{*}+\omega$ and $\theta(y)=\omega$; then

$$
\alpha \cdot \omega^{*}+\alpha \cdot \omega=\gamma \cdot \omega .
$$

Using 3.5 and the above equation, we cbtain either

(18) There exist nonzero types $\rho_{1}$ and $\rho_{2}$ such that $\alpha \cdot \omega^{*}=\gamma \cdot \rho_{1}$ and $\rho_{1}+\rho_{2}=\omega$, or

(19) There exist types $\rho_{1}, \rho_{2}, \gamma^{(1)}$ and $\gamma^{(2)}$ such that $\alpha \cdot \omega^{*}=\gamma \cdot \rho_{1}+\gamma^{(1)}$,

$$
\alpha \cdot \omega=\gamma^{(2)}+\gamma \cdot \rho_{2}, \rho_{1}+1+\rho_{2}=\omega, \gamma^{(1)}+\gamma^{(2)}=\gamma \text { and } \gamma^{(1)} \neq 0 \text {. }
$$

We note that (18) implies that $\rho_{1}$ is finite; now from (8) and the first equation of (18) we get (ii). If (19) holds, we must consider two cases, depending upon whether $\rho_{1}=0$ or $\rho_{1} \neq 0$. In the first case, from 3.5 , the second equation of (19) and the fact that $\rho_{2}$ cannot be zero, we obtain

(20) There exists a finite type $n$ and a type $\alpha^{(1)}$ such that $\gamma^{(2)}=\alpha \cdot n+\alpha^{(1)}$ and $\alpha^{(1)} \mathfrak{g} \alpha$.

It follows from (19) and (20) that

$$
\gamma=\gamma^{(1)}+\gamma^{(2)}=\alpha \cdot \omega^{*}+\alpha \cdot n+\alpha^{(1)}=\alpha \cdot \omega^{*}+\alpha^{(1)} ;
$$

thus (iii) holds. Now assume that $\rho_{1} \neq 0$; clearly, $\rho_{1}$ must be finite. We find, by using 3.5 and (19), that

(21) There is a type $\alpha^{(1)}$ such that $\alpha^{(1)} \mathfrak{g} \alpha$ and $\gamma \cdot \rho_{1}=\alpha \cdot \omega^{*}+\alpha^{(1)}$.

From (8) and (21), we again obtain (iii). Each of the other cases of (5) is symmetric with one of the two which have been proved in detail.

In the proof of 3.6 an essential use was made of the elementary decomposition property proved in 3.2. If we start with the hypothesis of 3.6 and if we employ, in place of 3.2, the scattered decomposition property of 3.3 , then we obtain Theorem 3.7 below; the method of proof is rather similar to that of 3.6 but is simpler. Note that 3.7 is an exact analogue of 3.6 in the sense that if we replace the words "finite" and "scattered" by "infinite" and "nonscattered" respectively everywhere in the statement of 3.6, we obtain 3.7.

TheOREm 3.7. If $\alpha \cdot \beta=\gamma \cdot \delta \neq 0$, then at least one of the following conditions is satisfied: 
(i) $\beta=\delta$,

(ii) There exist types $\gamma_{1}$ and $\gamma_{2}$ with $\gamma_{1} \mathcal{F} \gamma$ and $\gamma_{2} \mathfrak{g} \gamma$ and also a nonzero, nonscattered type $\delta^{\prime}$ such that $\alpha=\gamma_{1}+\gamma \cdot \delta^{\prime}+\gamma_{2}$ and $\delta^{\prime} \mathfrak{T T} \delta$,

(iii) There exist types $\alpha_{1}$ and $\alpha_{2}$ with $\alpha_{1} \mathfrak{F} \alpha$ and $\alpha_{2} \mathfrak{g} \alpha$ and also a nonzero, nonscattered type $\beta^{\prime}$ such that $\gamma=\alpha_{1}+\alpha \cdot \beta^{\prime}+\alpha_{2}$ and $\beta^{\prime} \mathfrak{T C} \beta$,

(iv) There exist scattered types $\gamma^{\prime}$ and $\delta^{\prime}$ such that $\alpha \cdot \beta^{\prime}=\gamma \cdot \delta^{\prime}, \beta^{\prime}$ IT $\beta$ and $\delta^{\prime} \mathfrak{T l} \delta$.

Proofs of the following two lemmas are known( $\left.{ }^{4}\right)$.

Lemma 3.8. For any types $\alpha, \beta, \gamma$, the conditions $\alpha=\beta+\alpha+\gamma$ and $\alpha=\beta+\alpha$ $=\alpha+\gamma$ are equivalent.

Lemma 3.9. If $n$ is a finite type and if $\alpha \cdot n+\beta=\alpha \cdot n+\gamma$, then $\alpha+\beta=\alpha+\gamma$.

We shall say that $\gamma$ is a left cancelling type if, for all types $\alpha$ and $\beta$, the equality $\gamma \cdot \alpha=\gamma \cdot \beta$ implies $\alpha=\beta$.

THEOREM 3.10. The following conditions are equivalent:

(i) $\boldsymbol{\gamma}$ is a left cancelling type,

(ii) $\boldsymbol{\gamma} \neq \boldsymbol{\gamma}+\boldsymbol{\gamma} \cdot \alpha+\gamma$ for every $\alpha$,

(iii) $\gamma \neq \gamma \cdot \alpha$ for every $\alpha \neq 1$.

Proof. Clearly (i) implies (ii). We shall show that (ii) implies (iii), and (iii) implies (i).

Assume that there is a type $\gamma$ which satisfies (ii) but not (iii). Then for some type $\beta$ we have

$$
\gamma=\gamma \cdot \beta \text { and } \beta \neq 1 .
$$

Since $\gamma$ satisfies (ii), $\gamma \neq 0$; therefore $\beta \neq 0$ and $\beta$ is representable in the form

$$
\beta=\beta^{\prime}+1+\beta^{\prime \prime}+1+\beta^{\prime \prime \prime} \text {. }
$$

Hence we have

$$
\gamma=\gamma \cdot \beta^{\prime}+\gamma+\gamma \cdot \beta^{\prime \prime}+\gamma+\gamma \cdot \beta^{\prime \prime \prime} .
$$

Applying 3.8 to the above equality twice, we obtain

$$
\gamma=\gamma+\gamma \cdot \beta^{\prime \prime}+\gamma=\gamma \cdot\left(1+\beta^{\prime \prime}+1\right),
$$

in contradiction to the assumption that $\gamma$ does not satisfy (iii).

Suppose finally that $\gamma$ satisfies (iii) but not (i). Then $\gamma \neq 0$ and there must exist types $\alpha$ and $\beta$ such that

$$
\gamma \cdot \alpha=\gamma \cdot \beta \text { and } \alpha \neq \beta .
$$

Using 3.6 and (1) we obtain either

(4) Lemmas 3.8 and 3.9 are stated without proof in Lindenbaum and Tarski [11]; for their proofs see Sierpiński $[13$, p. 5] and Tarski $[15$, p. 28] respectively. 
(2) There are types $\gamma_{1}$ and $\gamma_{2}$ and an infinite type $\delta$ such that $\gamma=\gamma_{1}+\boldsymbol{\gamma} \cdot \delta+\gamma_{2}$, or

(3) For some distinct finite nonzero types $m$ and $n, \boldsymbol{\gamma} \cdot m=\boldsymbol{\gamma} \cdot n$.

Using the fact that $\delta \notin\{0,1\}$, and applying 3.8 to (2) twice, we obtain

$$
\gamma=\gamma \cdot \delta^{\prime}, \text { where } \delta^{\prime} \neq 1,
$$

in contradiction to our assumption that $\gamma$ does not satisfy (iii). From (3) and 3.9 we also obtain (4).

Given types $\alpha$ and $\beta$, we write $\alpha \& \beta$ if there exist relations $R$ and $S$ such that $\tau(R)=\alpha, \tau(S)=\beta$ and $R$ is a subrelation of $S$ (cf. Fraissé [7]). If $\alpha \& \beta$ is false, we write $\alpha \bar{\alpha} \beta$.

The following lemma is an immediate consequence of the above definition.

LEMMA 3.11.

(i) If $\alpha £ \beta$ and $\beta £ \gamma$, then $\alpha £ \gamma$.

(ii) If $R$ is a relation, and if $\alpha$ and $\beta$ are functions on $F(R)$ such that $\alpha(x)<\beta(x)$ for each $x \in F(R)$, then $\sum_{R} \alpha(x) \prec \sum_{R} \beta(x)$.

(iii) If $\alpha £ \beta$, then $\gamma \cdot \alpha £ \gamma \cdot \beta$.

Lemma 3.12. If $\alpha$ is a scattered type, then $\alpha+\alpha \bar{\AA} \alpha$.

Proof. For the proof see Ginsburg [8, p. 519, Lemma 1.4] $\left(^{5}\right)$.

The converse of 3.12 does not hold; e.g., let $\alpha=\sum_{s} \lambda^{x}$, where $S$ is the usual ordering relation for the natural numbers. Then $\alpha$ is clearly dense, while on the other hand one can show that $\alpha+\alpha \overline{\mathscr{L}} \alpha$.

ThEOREM 3.13. Every scattered type is a left cancelling type.

Proof. This follows from 3.10, (i) and (iii), and 3.12.

Theorem 3.13 is a generalization of the result, due to Lindenbaum, that every nonzero ordinal (or inverse of an ordinal) is a left cancelling type ${ }^{(6)}$.

We say that the ordered pair $\langle\beta, \gamma\rangle$ is a gap of $\alpha$ if $\alpha$ is expressible in the form $\alpha=\beta+\gamma$, where $1 \overline{\mathfrak{F}} \beta, 1 \overline{\mathfrak{g}} \gamma, \beta \neq 0$ and $\gamma \neq 0$. An immediate consequence of this definition is the following lemma.

Lemma 3.14. If $\alpha \neq 0,1 \overline{\mathfrak{g}} \alpha, 1 \overline{\mathcal{F}} \alpha$ and $2 \mathfrak{L} \delta$, then $\alpha \cdot \delta$ has a gap.

THEOREM 3.15. If $\alpha$ is a nonzero type such that

(i) $\alpha$ has no gaps,

(ii) $1 \bar{g} \alpha$ and $1 \overline{\mathfrak{F}} \alpha$, then $\alpha$ is a left cancelling type.

(5) Actually in [8] a stronger result has been established; it is shown there that if $\alpha+\alpha \mathscr{L} \alpha$, then $\alpha \cdot \eta \mathfrak{L} \alpha$. Lemma 3.12 was proved independently (and at about the same time) by the author; see [1].

${ }^{(6)}$ Lindenbaum's result is stated without proof in Lindenbaum and Tarski $[11$, p. 321, Theorem 15]. A prorf can be found in Sierpinski [13]. 
Proof. By contradiction, using 3.10, (i) and (iii), and 3.14.

It follows from 3.15 that the type $\lambda$ of the real numbers is a left cancelling type. We note that Theorem 3.15 cannot be improved by weakening or omitting the hypothesis (ii), since $(1+\lambda) \cdot 2=1+\lambda,(\lambda+1) \cdot 2=\lambda+1$ and $(1+2 \cdot \lambda+1) \cdot 2=1+2 \cdot \lambda+1$.

4. Solutions of the equation $\xi^{n}=\alpha$.

THEOREM 4.1. If $\beta^{2}=\gamma^{2}$ and if

(i) $\beta$ has no gaps,

(ii) $1 \bar{g} \beta$ and $1 \bar{F} \beta$, then $\beta=\gamma$.

Proof. For $\beta=0$, the theorem is trivial. Suppose now that $\beta \neq 0$. Then, clearly, we have

$$
1 \overline{\mathfrak{g}} \gamma \text { and } 1 \overline{\mathfrak{F}} \gamma \text {. }
$$

Since $\beta \neq 0, \beta$ is expressible in the form

and hence

$$
\beta=\beta_{1}+1+\beta_{2}
$$

$$
\left(\beta \cdot \beta_{1}+\beta\right)+\beta \cdot \beta_{2}=\gamma \cdot \gamma \text {. }
$$

It follows from 3.5 and the above equality that at least one of the two following conditions must be satisfied:

(2) There exist types $\gamma_{1}$ and $\gamma_{2}$ such that $\beta \cdot \beta_{1}+\beta=\gamma \cdot \gamma_{1}$ and $\beta \cdot \beta_{2}=\gamma \cdot \gamma_{2}$.

(3) There exist nonzero types $\gamma^{(1)}$ and $\gamma^{(2)}$ as well as types $\gamma_{1}$ and $\gamma_{2}$ such that $\beta \cdot \beta_{1}+\beta=\gamma \cdot \gamma_{1}+\gamma^{(1)}, \beta \cdot \beta_{2}=\gamma^{(2)}+\gamma \cdot \gamma_{2}$ and $\gamma^{(1)}+\gamma^{(2)}=\gamma$.

If we apply 3.5 again to the first equality of (2), we obtain either

(4) There exists a type $\delta$ such that $\beta=\gamma \cdot \delta$,

or

(5) There are types $\gamma_{1,1}, \gamma_{1,2}, \gamma^{(1)}$ and $\gamma^{(2)}$ such that $\beta \cdot \beta_{1}=\boldsymbol{\gamma} \cdot \boldsymbol{\gamma}_{1,1}+\boldsymbol{\gamma}^{(1)}$, $\beta=\gamma^{(2)}+\gamma \cdot \gamma_{1,2}, \gamma^{(1)} \neq 0, \gamma^{(2)} \neq 0$ and $\gamma^{(1)}+\gamma^{(2)}=\gamma$.

Suppose that (4) holds. Then $\delta=1$, for if we had $2 \mathcal{L} \delta$, then, by 3.14 , $\beta$ would have a gap, contrary to hypothesis (i). Hence we have $\beta=\gamma$.

Assume now that (5) holds. First consider the case $\gamma_{1,2} \neq 0$. Using (1) and (5), we find the $1 \bar{F} \gamma^{(2)}$; using (1) again, we obtain $1 \overline{\mathscr{T}} \boldsymbol{\gamma} \cdot \boldsymbol{\gamma}_{1,2}$. The conclusion that $\beta$ must have a gap follows from (5). If $\gamma_{1,2}=0$, we apply 3.5 again to the first equality of (5) and obtain at least one of the following two conditions:

There is a nonzero type $\theta$ such that $\gamma^{(1)}=\beta \cdot \theta$.

(7) There are types $\beta_{1,1}, \beta_{1,2}, \beta^{(1)}$ and $\beta^{(2)}$ such that $\gamma \cdot \gamma_{1,1}=\beta \cdot \beta_{1,1}+\beta^{(1)}$,

$$
\gamma^{(1)}=\beta^{(2)}+\beta \cdot \beta_{1,2}, \beta^{(1)}+\beta^{(2)}=\beta, \beta^{(1)} \neq 0 \text { and } \beta^{(2)} \neq 0 \text {. }
$$


From (5), (6) and the assumption that $\gamma_{1,2}=0$ it follows that

$$
\gamma=\gamma^{(1)}+\gamma^{(2)}=\beta \cdot \theta+\beta=\beta \cdot(\theta+1),
$$

with $\beta_{1,2} \neq 0$. Hence $\gamma$ is expressible in the form

$$
\gamma=\beta \cdot \epsilon, \quad \text { where } 2 \& \epsilon .
$$

We have then

$$
\gamma^{2}=\beta^{2}=\beta \cdot \epsilon \cdot \beta \cdot \epsilon,
$$

and since, by $3.15, \beta$ is a left cancelling type, we obtain

$$
\beta=\epsilon \cdot(\beta \cdot \epsilon) \text {. }
$$

By $3.14, \beta \cdot \epsilon$ must have a gap; from this fact and (9) it follows readily that $\beta$ must also have a gap, in contradiction to (i).

For the case (7), we see, using (1) and (5), that $1 \overline{\mathscr{F}} \beta^{(1)}$ and $1 \bar{g} \beta^{(2)}$; hence $\beta$ must have a gap.

We still have to consider (3). From 3.4 and the first identity of (3), we obtain either

(10) There is a $\delta$ such that $\beta \cdot \beta_{1}=\gamma \cdot \gamma_{1}+\delta$ and $\gamma^{(1)}=\delta+\beta$,

or

(11) There is a $\delta$ such that $\gamma \cdot \gamma_{1}=\beta \cdot \beta_{1}+\delta$ and $\beta=\delta+\gamma^{(1)}$.

From 3.5 and the first identity of (10) we get at least one of the following conditions:

(12) There are types $\beta_{1,1}$ and $\beta_{1,2}$ such that $\gamma \cdot \gamma_{1}=\beta \cdot \beta_{1,1}$ and $\delta=\beta \cdot \beta_{1,2}$.

(13) There are types $\beta_{1,1}, \beta_{1,2}, \beta^{(1)}$ and $\beta^{(2)}$ such that $\gamma \cdot \gamma_{1}=\beta \cdot \beta_{1,1}+\beta^{(1)}$, $\delta=\beta^{(2)}+\beta \cdot \beta_{1,2}, \beta^{(1)} \neq 0, \beta^{(2)} \neq 0$ and $\beta^{(1)}+\beta^{(2)}=\beta$.

Using 3.5 and the second identity of (3), we obtain either

(14) There exists a type $\beta^{\prime}$ such that $\gamma^{(2)}=\beta \cdot \beta^{\prime}$,

or

(15) There are types $\beta_{2,1}, \beta_{2,2}, \beta^{(3)}$ and $\beta^{(4)}$ such that $\gamma^{(2)}=\beta \cdot \beta_{2,1}+\beta^{(3)}$, $\gamma \cdot \gamma_{2}=\beta^{(4)}+\beta \cdot \beta_{2,2}, \beta^{(3)} \neq 0, \beta^{(4)} \neq 0$ and $\beta^{(3)}+\beta^{(4)}=\beta$.

Suppose that (12) and (14) hold. From (3), (10), (12) and (14), we get $\gamma=\gamma^{(1)}+\gamma^{(2)}=(\delta+\beta)+\beta \cdot \beta^{\prime}=\left(\beta \cdot \beta_{1,2}+\beta\right)+\beta^{\prime}=\beta \cdot\left(\beta_{1,2}+1+\beta^{\prime}\right)$. By (3) and (14), $\beta_{2,1} \neq 0$. Hence we obtain (8); but we have already proved that (8) cannot hold.

We recall (1): $1 \overline{\mathcal{g}} \gamma$ and $1 \overline{\mathcal{F}} \gamma$. Using (13), (10) and (3) successively, we get $\beta^{(2)} \mathfrak{g} \delta, \delta \mathfrak{g} \gamma^{(1)}$ and $\gamma^{(1)} \mathfrak{g} \gamma$; consequently $1 \bar{g} \beta^{(2)}$. Moreover, the first 
equality in (13) shows that $1 \bar{F} \beta^{(1)}$. The last three statements of (13) now show that $\beta$ must have a gap, in contradiction to (i).

If $\delta=0$ in (11), then, by (3), (11) and (14), we have

$$
\gamma=\gamma^{(1)}+\gamma^{(2)}=\beta+\beta \cdot \beta^{\prime}=\beta \cdot\left(1+\beta^{\prime}\right) \text { and } \beta^{\prime} \neq 0 ;
$$

this implies (8). If $\delta \neq 0$, then, using (11) and 3.14, we obtain $1 \bar{F} \delta$; since $1 \bar{g} \gamma^{(1)}$, the last equation of (11) shows that $\beta$ must have a gap.

Finally, suppose that (15) holds; from (15) and the fact that $1 \bar{g} \gamma \cdot \gamma^{(2)}$ and $1 \bar{F} \gamma^{(2)}$, we see that $\beta$ must have a gap. This completes the proof.

Lemma 4.2. For every type $\alpha$ and for every positive integer $n$ the following conditions are equivalent:

(i) $\alpha+\alpha £ \alpha$,

(ii) $\alpha^{n}+\alpha^{n} \mathscr{L} \alpha^{n}$.

Proof. From 3.11 (iii), we see that (i) implies (ii).

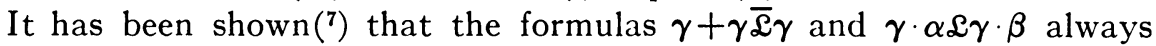
imply $\alpha \overline{\mathcal{L}} \beta$. Now assume that there is a positive integer $n$ such that $\alpha^{n}+\alpha^{n}$ $\mathscr{L} \alpha^{n}$, while $\alpha+\alpha \overline{\mathcal{L}} \alpha$. Then $2 \mathfrak{L} n$ and $\alpha \cdot\left(\alpha^{n-1}+\alpha^{n-1}\right) \mathscr{L} \alpha \cdot \alpha^{n-1}$; but by an obvious induction we obtain $\alpha+\alpha £ \alpha$.

Theorem 4.3. Suppose that $\alpha$ is a type such that $\alpha+\alpha £ \alpha$ and that $n$ is a positive integer. Then the equation

has at most one solution.

$$
\xi^{n}=\alpha
$$

Proof. By induction. The case $n=1$ is trivial. Assume that the theorem holds for some positive $n$ and that there are types $\beta$ and $\gamma$ such that

$$
\beta^{n+1}=\gamma^{n+1}=\alpha .
$$

By (1) and 4.2 we have

$$
\beta+\beta \overline{\mathfrak{L}} \beta .
$$

If we apply 3.6 to the identity $\beta \cdot \beta^{n}=\gamma \cdot \gamma^{n}$, we obtain at least one of the four following conditions:

$$
\beta^{n}=\gamma^{n} \text {. }
$$

(4) There is an infinite type $\gamma^{\prime}$ such that $\gamma \cdot \gamma^{\prime} \& \beta$.

(5) There is an infinite type $\beta^{\prime}$ such that $\beta \cdot \beta^{\prime} \& \gamma$.

(6) There are distinct finite types $m$ and $p$ such that $\beta \cdot m=\gamma \cdot p$.

If (3) holds, then by the inductive premise we get $\beta=\gamma$. Suppose that (4) holds. Then

(7) See Ginsburg $[9$, p. 564, Theorem 7]. Lemma 4.2 was originally proved by the author independently of [9] (and actually before [9] appeared in print; see [3]). 


$$
\gamma+\gamma \mathscr{L} \gamma \cdot \gamma^{\prime} \mathfrak{L} \beta
$$

now from (1) we obtain

$\alpha+\alpha=\gamma^{n+1}+\gamma^{n+1}=\gamma^{n} \cdot(\gamma+\gamma)$

$$
\mathfrak{L}(\gamma+\gamma)^{n} \cdot(\gamma+\gamma)=(\gamma+\gamma)^{n+1} \mathfrak{L} \beta^{n+1}=\alpha,
$$

in contradiction to (1). The case (5) is symmetric with (4).

In order to derive our conclusion in case (6), we define a function $H$ on all nonzero types $\delta$ into the class of finite types as follows: Let $\sum_{R} \phi(x)$ be an elementary decomposition of $\delta$. If $\phi(x)$ is infinite for each $x \in F(R)$, we put $H(\delta)=0$. If $\phi(x)$ is finite for some $x \in F(R)$, we put $H(\delta)=p$, where $p$ is the unique type such that $\phi(x)=p$ for some $x \in F(R)$ while $p \& \phi(x)$ for every $x \in F(R)$. We shall show that the function $H$ has the following properties:

(7) If $\delta$ is an infinite type and if $q$ is a positive integer, then $H(\delta)=H\left(\delta^{q}\right)$.

(8) $H(p \cdot \delta)=p \cdot H(\delta)$ for every nonzero finite type $p$.

To prove (7), we first note that the formulas $H(\delta)=0$ and $H\left(\delta^{q}\right)=0$ are clearly equivalent.

Suppose now that $\delta$ is infinite, that $H(\delta)=p$, with $1 \& p$, and that $q$ is a positive integer. Then, by $3.2, \delta$ is representable in one of the following forms: (a) $\delta=p+\epsilon$, where $\epsilon \neq 0$ and $1 \bar{g} \epsilon$, (b) $\delta=\epsilon+p$, where $\epsilon \neq 0$ and $1 \bar{F} \epsilon$, (c) $\delta$ $=\epsilon+p+\gamma$, where $\epsilon \neq 0, \gamma \neq 0,1 \bar{F} \epsilon$ and $1 \bar{g} \gamma$. If (a) holds, then $1 \mathfrak{g} \delta$; consequently $1 \mathfrak{g} \delta^{q-1}$. Hence

and

$$
\delta^{q}=\delta \cdot\left(1+\delta^{\prime}\right), \text { where } 1+\delta^{\prime}=\delta^{q-1},
$$

$$
\delta^{q}=p+\epsilon+\delta \cdot \delta^{\prime}
$$

From this, we see that

$$
H\left(\delta^{q}\right) \mathscr{L} H(\delta) .
$$

The case (b) is similar to (a). If (c) holds, then we have $(\epsilon+p+\gamma) \mathfrak{M} \delta^{a}$, and again we obtain (9).

Now assume that $H\left(\delta^{q}\right)=p$, with $1 \mathscr{L} p$, and that $q$ is a positive integer. Then $\delta^{q}$ is of the form

$$
\delta^{q}=\delta \cdot \delta^{q-1}=(\epsilon+p)+\gamma, \text { where } 1 \overline{\mathcal{F}} \epsilon \text { and } 1 \bar{g} \gamma .
$$

If we apply 3.5 to (10), we obtain either

(11) There is a type $\phi$ such that $\epsilon+p=\delta \cdot \phi$,

or

(12) There are types $\delta_{1}, \delta_{2}, \delta^{(1)}$ and $\delta^{(2)}$ such that $\epsilon+p=\delta \cdot \delta_{1}+\delta^{(1)}$,

$$
\gamma=\delta^{(2)}+\delta \cdot \delta_{2}, \delta^{(1)}+\delta^{(2)}=\delta \text { and } \delta^{(1)} \neq 0 \text {. }
$$


Applying 3.5 again to the first equality of (11), and recalling that $\delta$ is infinite while $p$ is finite, we get

(13) There exist types $\delta_{3}$ and $\delta^{(3)}$ such that $\epsilon=\delta \cdot \delta_{3}+\delta^{(3)}, \delta^{(3)}+p=\delta$ and $\delta^{(3)} \neq 0$.

It follows from (10) and (13) that $H(\delta) \& p$ and hence

$$
H(\delta) \mathfrak{L} H\left(\delta^{q}\right) .
$$

If we apply 3.4 to the first identity of (12), we get either

(15) There is a type $\zeta$ such that $\epsilon=\delta \cdot \delta_{1}+\zeta$ and $\delta^{(1)}=\zeta+p$,

or

(16) There is a type $\theta$ such that $p=\theta+\delta^{(1)}$.

Using (10) and (12), we find that $1 \bar{g} \delta^{(2)}$. If (15) holds, we also obtain $\delta=\zeta+p+\delta^{(2)}$, while it follows from (10) and (15) that $1 \overline{\mathcal{F}} \zeta$; hence (14) is valid. If (16) holds, then $\delta^{(1)}$ is finite, in fact $1 \& \delta^{(1)} \& p$; now from the equation $\delta=\delta^{(1)}+\delta^{(2)}$ we obtain (14). This completes the proof of (7).

To prove (8), one need only note that if $\sum_{R} \phi(x)$ is an elementary decomposition of $\delta$, then $\sum_{R}(p \cdot \phi(x))$ is an elementary decomposition of $p \cdot \delta$.

Now from (6), using two results of Lindenbaum $\left({ }^{8}\right)$, we obtain the existence of a type $\delta$ such that

(17) $\beta=\delta \cdot p^{\prime}$ and $\gamma=\delta \cdot m^{\prime}$, where $m^{\prime}$ and $p^{\prime}$ are distinct finite types.

It follows from (1) that (17) can hold only when $\beta$ is infinite.

Suppose that $\delta$ is a left cancelling type. By (1) and (17) we have

$$
p^{\prime} \cdot \beta^{n}=m^{\prime} \cdot \gamma^{n}
$$

while, by (1) and (7),

$$
H(\beta)=H(\gamma)=H\left(\beta^{n}\right)=H\left(\gamma^{n}\right) .
$$

If $H(\beta)=0$, then $k \cdot \beta=\beta$ for finite $k$ (as is shown at once by consideration of the possible infinite elementary types); hence, using (18), we get

$$
\beta^{n}=p^{\prime} \cdot \beta^{n}=m^{\prime} \cdot \gamma^{n}=\gamma^{n},
$$

and from the inductive premise we conclude that $\beta=\gamma$. If $H(\beta) \neq 0$, it follows from (8), (18) and (19) that

$$
p^{\prime} \cdot H(\beta)=m^{\prime} \cdot H(\beta),
$$

which is impossible.

(8) The results, Theorems 13 and 14 of [11], are as follows: (1) If $\alpha \cdot n=\beta \cdot n$, where $n$ is a nonzero finite type, then $\alpha=\beta$; (2) If $\alpha \cdot m=\beta \cdot n$, where $m$ and $n$ are relatively prime, then there exists a type $\gamma$ such that $\alpha=\gamma \cdot n$ and $\beta=\gamma \cdot m$. 
Finally, assume that $\delta$ is not a left cancelling type. By 3.10, (i) and (iii), $\delta$ is of the form

$$
\delta=\delta \cdot \epsilon, \text { where } 2 \& \epsilon ;
$$

from (17), (20) and 3.11, (ii) and (iii), we get

$$
\beta+\beta=\delta \cdot p^{\prime} \cdot 2=\delta \cdot 2 \cdot p^{\prime} \& \delta \cdot \epsilon \cdot p^{\prime}=\delta \cdot p^{\prime}=\beta,
$$

in contradicton to (2).

Corollary 4.4. Let $\alpha$ be any scattered type and let $n$ be a positive integer. Then the equation $\xi^{n}=\alpha$ has at most one solution.

Proof. This is a consequence of 3.12 and 4.3.

Corollary 4.4 is a generalization of the well known result $\left({ }^{9}\right)$ that $\xi^{n}=\alpha$ has at most one solution when $\alpha$ is an ordinal.

We now consider the problem of the existence of types $\alpha$ for which the equation $\xi^{n}=\alpha$, and in particular $\xi^{2}=\alpha$, has more than one solution( $\left.{ }^{10}\right)$. If such a type $\alpha$ exists, it must, by 4.4 , be nonscattered. Hence it is natural to ask first whether such types can be found in the class of denumerable nonscattered types; the answer turns out to be positive.

As is well known, there are exactly four denumerable dense types, namely $\eta, 1+\eta, \eta+1$ and $1+\eta+1$. An obvious consequence of this fact is the following lemma.

Lemma 4.5. Let $\alpha$ be any nonzero denumerable type. Then

(i) $\eta \cdot \alpha=\eta$,

(ii) If $1 \mathcal{F} \alpha$, then $(\eta+1) \cdot \alpha=\eta$,

(iii) If $1 \bar{g} \alpha$, then $(1+\eta) \cdot \alpha=\eta$.

Suppose that $\alpha$ is a nonscattered denumerable type other than 0 . We see from 3.3 that $\alpha$ is representable in the form $\alpha=\sum_{R} \gamma(x)$, where the types $\gamma(x)$ are scattered and denumerable, while $\tau(R)$ is one of the four denumerable dense types. Among all nonscattered denumerable types, clearly the simplest are the types for which all the $\gamma(x)$ are equal; among these can be found a type having more than one "square root." Indeed, the type $\omega \cdot \eta$ has this property $\left({ }^{11}\right)$. For, it follows from 4.5 (i) that

$$
(\omega \cdot \eta)^{2}=\omega \cdot(\eta \cdot \omega \cdot \eta)=\omega \cdot \eta \cdot \eta=\omega \cdot \eta .
$$

Also, we have, by 4.5 , (i) and (ii),

(9) See e.g. Sierpiłski [12, p. 189].

(10) The problem is not new, although it appears that it was stated in print for the first time in [2]. According to the information available, it originated with Cantor.

(11) The first example of a type $\alpha$ for which the equation $\xi^{2}=\alpha$ has more than one solution was given by the author in [2]. Later Sierpinski simplified the example and in fact showed that $\omega \cdot \eta$ can be taken for $\alpha$; see [6]. 


$$
\omega \cdot(\eta+1)^{2}=\omega \cdot \eta \text {. }
$$

On the other hand, since $\omega$ is, by 3.13 , a cancelling type, we have

$$
\omega \cdot \eta \neq \omega \cdot(\eta+1) \text {. }
$$

It can easily be shown that $\omega \cdot \eta$ and $\omega \cdot(\eta+1)$ are the only solutions of the equation $\xi^{2}=\omega \cdot \eta$. Moreover, a denumerable scattered type $\alpha$ can be chosen so that $\xi^{2}=\alpha \cdot \eta$ has exactly $\mathfrak{m}$ solutions, where $1 \leqq \mathfrak{m} \leqq \boldsymbol{N}_{0}$. It seems extremely likely that for $3<\mathfrak{m}<\boldsymbol{\aleph}_{0}$ there are only finitely many suitable types $\alpha$, and it is known that no such type $\alpha$ exists for $\mathfrak{m}>\boldsymbol{\aleph}_{0}$. As will be seen in 4.12 , we obtain more complete results if we turn from types of the form $\alpha \cdot \eta$ to arbitrary nonscattered types $\left({ }^{12}\right)$.

From now on, we will denote the (usual) ordering relation of the rationals by the symbol $Q$. For convenience we now define:

$$
\begin{aligned}
\Delta & =\{\alpha ; \alpha \text { is a nonzero denumerable type }\}, \\
\Delta_{s} & =\{\alpha ; \alpha \in \Delta \text { and } \alpha \text { is scattered }\}, \\
\Delta_{\mathbf{s}^{\prime}} & =\left\{\alpha ; \alpha \in \Delta_{s}, 1 \mathfrak{g} \alpha \text { and } 1 \overline{\mathcal{F}} \alpha\right\}, \\
\Delta_{\mathbf{s}^{\prime \prime}} & =\left\{\alpha ; \alpha \in \Delta_{\boldsymbol{s}}, 1 \mathrm{~g} \alpha \text { and } 1 \mathfrak{F} \alpha\right\} .
\end{aligned}
$$

Lemma 4.6. Each of the sets $\Delta, \Delta_{s}, \Delta_{s^{\prime}}$, and $\Delta_{s^{\prime \prime}}$ is of power $2^{N_{0}}$.

Proof. As is known, every denumerable relation is isomorphic to a subrelation of $Q$. Hence the power of $\Delta$ is at most $2 \aleph_{0}$. On the other hand, we see from 3.2 that there are $2^{\aleph_{0}}$ distinct types $\alpha$ belonging to $\Delta_{s}$ of the form $\alpha=\sum_{s}\left(\omega^{*}+\omega+\phi(x)\right)$, where $\tau(S)=\omega$ and $\phi(x) \in\{0,1\}$. Consequently, there are also $2^{\aleph_{0}}$ distinct types of the form $1+\alpha$ and $2^{\aleph_{0}}$ distinct types of the form $1+\alpha+1$.

Lemma 4.7. If $a \in \Delta$ but $a \notin \Delta_{s}$, then $\alpha$ is representable in exactly one of the ollowing forms:

(i) $\alpha=\sum_{Q} \gamma(r)$,

(ii) $\alpha=\beta+\sum_{Q} \gamma(r)$,

(iii) $\alpha=\sum_{Q} \gamma(r)+\delta$,

(iv) $\alpha=\beta+\sum_{\Theta} \gamma(r)+\delta$;

where $\beta, \delta$ and each $\gamma(r)$ belong to $\Delta_{s}$.

Proof. This is immediate from 3.3.

We shall say that the set $A$ is dense in the relation $S$ if $A \subseteq F(S)$, and if every nonempty open interval of $S$ contains an element of $A$. The following theorem concerning dense subsets of $Q$ is known from the literature (see Skolem $[14$, p. 32]).

Theorem 4.8. Let

(i) $\mathrm{m}$ be any cardinal between 1 and $\boldsymbol{\aleph}_{0}$ inclusive.

(12) Detailed results of the discussion of the equation $\xi^{n}=\alpha \cdot \eta$ (where $\alpha$ is a denumerable scattered type) are stated without proof in [5] 
(ii) $A_{0}, A_{1}, \cdots, A_{i}, \cdots$ (where $0 \leqq i<\mathfrak{m}$ ) be a partition of the rational numbers, each $A_{i}$ being dense in $Q$.

(iii) $B_{0}, B_{1}, \cdots, B_{i}, \cdots$ (where $0 \leqq i<m$ ) be a partitiou of the rational numbers, each $B_{i}$ being dense in $Q$.

Then there exists an automorphism, $f$, of $Q$ onto $Q$, such that, for each $i, f$ maps the set $A_{i}$ onto $B_{i}$.

CoRollary 4.9. Suppose that $\mathrm{A} \subseteq \Delta_{8}$ and $1 \leqq \kappa(A) \leqq \boldsymbol{\aleph}_{0}$. Then there exists exactly one type $\alpha$ satisfying the conditions:

(i) $\alpha=\sum_{Q} \phi(r)$, where $\phi(r) \in \mathrm{A}$ for each $r \in F(Q)$.

(ii) For every type $\delta \in \mathrm{A},\{r ; r \in F(Q)$ and $\phi(r)=\delta\}$ is dense in $Q$.

Proof. $\left({ }^{13}\right)$ This follows from 3.3 and 4.8 .

For each $\mathrm{A} \subseteq \Delta_{\mathrm{s}}$ with $1 \leqq \kappa(A) \leqq \boldsymbol{\aleph}_{0}$, we shall denote the unique type $\alpha$ characterized in 4.9 by the symbol $\sigma(\mathrm{A})$.

LemMA 4.10. Let $\beta$ be any type and let $m$ and $n$ be any integers with $1 \leqq m \leqq n$. Then $\beta^{m} \mathfrak{T C} \beta^{n}$.

Proof. Obvious.

Lemma 4.11. Suppose that $\mathrm{A} \subseteq \Delta_{s}, 1 \leqq \kappa(A) \leqq \boldsymbol{\aleph}_{0}, \beta$ T $\sigma(\mathrm{A})$ and $\beta \notin \Delta_{s}$. Then there exist types $\gamma, \delta \in \mathrm{A}$ and also types $\gamma^{\prime}, \delta^{\prime}$ such that

$$
\beta=\gamma^{\prime}+\sigma(\mathrm{A})+\delta^{\prime}, \quad \gamma^{\prime} \mathfrak{F} \gamma \quad \text { and } \quad \delta^{\prime} \mathfrak{g} \delta .
$$

Proof. This is a simple consequence of 4.7 and 4.9.

We now state and prove the main theorem of this section.

THEOREM 4.12. If $n$ is an integer with $2 \leqq n$, and if $\mathfrak{m}$ is a finite cardinal, or if $\mathfrak{m}=\boldsymbol{\aleph}_{0}$, or if $\mathfrak{m}=2^{\aleph_{0}}$, then there exist $2^{\aleph_{0}}$ distinct denumerable nonscattered types $\alpha$ for which the equation

(i) $\xi^{n}=\alpha$

has exactly $\mathrm{m}$ solutions.

CASE I. $\mathfrak{m}=0$.

If $\beta$ and $\beta^{\prime}$ are distinct elements of $\Delta_{s}$, then $\eta+\beta \neq \eta+\beta^{\prime}$. It follows from 4.6 that there are $2^{\aleph_{0}}$ distinct types $\alpha$ of the form

$$
\alpha=\eta+\beta, \text { where } \beta \in \Delta_{s} \text { and } 2 \& \beta .
$$

Suppose that $\alpha$ satisfies (1) and that $\xi$ is a solution of the equation (i). Clearly $\xi$ cannot be scattered, since $\xi^{n}$ is not scattered. Using 4.10 , we see that either (a) $\xi=\eta$, or (b) there is a type $\beta^{\prime} \neq 0$ such that $\xi=\eta+\beta^{\prime}$ and $\beta^{\prime} \mathfrak{g} \beta$. But if (a) holds, then $\xi^{n}=\eta \neq \eta+\beta$. Assume that (b) holds. If $\beta^{\prime}=1$, then, from 4.5 (ii), we get that $\xi^{n}=\eta+1 \neq \eta+\beta$. Finally, suppose that $\xi=\eta+\beta^{\prime}$ and $2 \& \beta^{\prime}$;

${ }^{(13)}$ Corollary 4.9 was pointed out to me by Tarski, who used it originally for other purposes. 
as $\beta^{\prime}$ is scattered, we must have $2 \mathbb{T} \beta^{\prime}$. Hence $2 \mathfrak{T} \xi$, so that $\xi \cdot 2 \mathfrak{N} \xi^{2}$; since, by $4.10, \xi^{2} \mathfrak{T} \alpha$, we see that

$$
\left(\eta+\beta^{\prime}+\eta+\beta^{\prime}\right) \mathfrak{M}\left(\eta+\beta^{\prime}\right),
$$

in contradiction to 3.3 .

CASE II. $m=1$.

We shall show that for each of the continuously many types $\alpha$ of the form

$$
\alpha=\beta \cdot \eta \text { and } \beta \in \Delta_{8^{\prime \prime}},
$$

the equation (i) has the unique solution $\xi=\alpha$. It follows from 4.5(i) that $\alpha$ is actually a solution. Now suppose that $\xi$ is any solution. Evidently $\xi$ is not scattered. Now, taking $A=\{\beta\}$, we have that $\sigma(A)=\beta \cdot \eta$; and 4.10 shows that $\xi \mathfrak{T C} \beta \cdot \eta$. Applying 4.11 , we see that $\xi$ is representable in the form

$$
\xi=\beta^{\prime}+\beta \cdot \eta+\beta^{\prime \prime}, \text { where } \beta^{\prime} \mathfrak{F} \beta \text { and } \beta^{\prime \prime} \mathfrak{g} \beta .
$$

Assume that $\beta^{\prime \prime} \neq 0$. Then, by (4), $1 \mathfrak{F} \beta^{\prime}$, so that $\xi \mathcal{F} \xi \cdot \beta^{\prime}$. Now by 4.10 we have $\xi^{2} \mathfrak{M} \beta \cdot \eta$. Since $\xi^{2}=\xi \cdot \beta^{\prime}+\xi \cdot \beta \cdot \eta+\xi \cdot \beta^{\prime \prime}$, it follows that $(\xi+\xi \cdot \beta \cdot \eta) \mathfrak{M} \beta \cdot \eta$, that is

$$
\left(\beta^{\prime}+\beta \cdot \eta+\beta^{\prime \prime}+\xi \cdot \beta \cdot \eta\right) \mathfrak{M} \beta \cdot \eta .
$$

From (5) and 3.3 , we see that

$$
\beta^{\prime \prime}=\beta \quad \text { or } \quad \beta^{\prime \prime}=0 .
$$

But if $\beta^{\prime \prime}=\beta$, then it follows from (3) and (4) that $1 \mathcal{F} \xi$ and so $1 \mathcal{F} \beta \cdot \eta$, which is absurd. Therefore $\beta^{\prime \prime}=0$ and

$$
\xi=\beta^{\prime}+\beta \cdot \eta \text {. }
$$

If $\beta^{\prime}=1$, then $1 \mathfrak{g} \xi$ and so $1 \mathfrak{g} \beta \cdot \eta$, which is obviously false. Therefore $2 \& \beta^{\prime}$, and, since $\beta^{\prime}$ is scattered, $2 \mathfrak{M} \beta^{\prime}$. Hence $2 \mathfrak{T} \xi$ and $\xi \cdot 2 \mathfrak{T} \xi^{2}$. Moreover, by $4.10, \xi^{2} \mathfrak{N} \beta \cdot \eta$. Thus we have $\xi \cdot 2 \mathfrak{M} \beta \cdot \eta$ and consequently

$$
\left(\beta \cdot \eta+\beta^{\prime}+\beta \cdot \eta\right) \mathfrak{M} \beta \cdot \eta \text {. }
$$

It follows from (6) and 3.3 that

$$
\beta^{\prime}=\beta ;
$$

using (3) and (4), we again obtain $1 \mathfrak{g} \xi$, but, as was noted above, this is impossible. We may now conclude that our assumption that $\beta^{\prime} \neq 0$ was incorrect. A symmetric argument shows that $\beta^{\prime \prime}=0$.

CASE III. $2 \leqq \mathfrak{m} \leqq \boldsymbol{N}_{0}$.

Let $\alpha$ be one of the continuum of types such that

$$
\alpha=\sigma(\mathrm{A}), \text { where } \mathrm{A} \subseteq \Delta_{8^{\prime}} \text { and } \kappa(A)=\mathfrak{m}-1 .
$$

(It is assumed here that $\boldsymbol{\aleph}_{0}-1=\boldsymbol{\aleph}_{0}$.) 
We make use of the following consequence of 4.7 and 4.9:

(8) If $\beta \in \mathrm{A}$ or if $\beta=0$, and if $\gamma \in \Delta$ and $1 \bar{F} \gamma$, then $(\sigma(\mathrm{A})+\beta) \cdot \gamma=\sigma(\mathrm{A})$.

Now we put

$$
\xi=\sigma(\mathrm{A})+\beta, \text { where } \beta \in \mathrm{A} \text { or } \beta=0 \text {. }
$$

Then, by (8),

$$
\xi^{2}=(\sigma(\mathrm{A})+\beta) \cdot(\sigma(\mathrm{A})+\beta)=\sigma(\mathrm{A})=\xi^{n}=\alpha .
$$

Now suppose that $\xi$ is a solution of (i). From 4.10 and 4.11 , we obtain the existence of types $\beta, \beta^{\prime}, \gamma$ and $\gamma^{\prime}$ such that

$$
\xi=\beta^{\prime}+\sigma(\mathrm{A})+\gamma^{\prime}, \quad \text { where } \beta^{\prime} \mathfrak{F} \beta, \gamma^{\prime} \mathfrak{g} \gamma \text { and } \beta, \gamma \in \mathrm{A} \text {. }
$$

First, suppose that $\gamma^{\prime} \neq 0$. By (7) and (10), $1 \mathfrak{g} \gamma^{\prime}$ and hence $\xi \mathfrak{g} \xi \cdot \gamma^{\prime}$. We know from 4.10 that $\xi^{2} \mathfrak{T} \sigma(\mathrm{A})$. Since $\xi^{2}=\xi \cdot \beta^{\prime}+\xi \cdot \sigma(\mathrm{A})+\xi \cdot \gamma^{\prime}$, it follows that $\left(\xi \cdot \sigma(\mathrm{A})+\xi \cdot \gamma^{\prime}\right) \mathfrak{N} \sigma(\mathrm{A})$ and consequently

$$
\left(\xi \cdot \sigma(\mathrm{A})+\beta^{\prime}+\sigma(\mathrm{A})\right) \mathfrak{M} \sigma(\mathrm{A}) .
$$

By (11), 3.3 and the definition of $\sigma(\mathrm{A})$, we have

$$
\beta^{\prime} \in \mathrm{A} \text { or } \beta^{\prime}=0 .
$$

If $\beta^{\prime} \in \mathrm{A}$, then, by (7), $1 \mathscr{I} \beta^{\prime}$; hence $1 \mathscr{I} \xi$ and $1 \mathscr{g} \sigma(\mathrm{A})$; but it is obvious that

$$
1 \bar{g} \sigma(\mathrm{A}) \text {. }
$$

Thus $\xi$ must be of the form

$$
\xi=\sigma(\mathrm{A})+\gamma^{\prime}
$$

Clearly $\gamma^{\prime} \neq 1$; since $\gamma^{\prime}$ must be scattered, we have $2 \Re \gamma^{\prime}, 2 \Re \xi$, and consequently $\xi \cdot 2$ IN $\xi^{2}$. Now using 4.10 , we obtain

$$
\left(\sigma(\mathrm{A})+\gamma^{\prime}+\sigma(\mathrm{A})\right) \mathfrak{M} \sigma(\mathrm{A}) \text {. }
$$

Applying 3.3 to (13), we find that $\gamma^{\prime} \in A$, and so $\xi$ satisfies (9).

Second, suppose that $\gamma^{\prime}=0$. If $\beta^{\prime}=0$, then $\xi$ obviously satisfies (9). Consider the case $\beta^{\prime} \neq 0$. We cannot have $\beta^{\prime}=1$, for then we could derive 1 g $\sigma(A)$, in contradiction to (12). Hence $2 \mathcal{L} \beta^{\prime}, 2 \mathfrak{N} \beta^{\prime}$ and $2 \mathfrak{N} \xi$. Now, with the help of 4.10 , we find that

$$
\left(\sigma(\mathrm{A})+\beta^{\prime}+\sigma(\mathrm{A})\right) \mathfrak{M} \sigma(\mathrm{A}) .
$$

From (14) and 3.3, we obtain

$$
\beta^{\prime} \in \mathrm{A}
$$

but an obvious consequence of $\beta^{\prime} \in \mathrm{A}$ is

$$
1 \mathscr{g} \sigma(\mathrm{A}) \text {, }
$$


which contradicts (12); hence the case $\beta^{\prime} \neq 0$ is impossible. We now conclude that (9) gives all solutions of (i).

CASE IV. $\mathfrak{m}=2^{\boldsymbol{k}_{0}}$.

We put

(15) $\epsilon=\sum_{Q} \gamma(r)$, where $\gamma$ is a one-to-one function on $F(Q)$ into $\Delta_{s}$.

Now choose $\alpha$ of the form

$$
\alpha=\left(\omega^{*}+\omega\right) \cdot \epsilon \cdot \eta .
$$

(In particular, in selecting $\epsilon$ we may take the types $\gamma(r)$ of (15) to be distinct nonzero finite types.) It follows from 3.3 and 3.13 that there are $2^{\aleph_{0}}$ types $\alpha$ satisfying (16).

Suppose that $\xi$ is a type such that

$$
\xi=\left(\omega^{*}+\omega\right) \cdot(\beta+\epsilon \cdot \eta+\delta) \text {, where } \delta+\beta \in\{0, \epsilon\} .
$$

Since

$$
(\beta+\epsilon \cdot \eta+\delta) \cdot\left(\omega^{*}+\omega\right)=\cdots+\beta+\epsilon \cdot \eta+\delta+\beta+\epsilon \cdot \eta+\delta+\cdots=\epsilon \cdot \eta,
$$

we find, using $4.5(i)$, that

$$
\xi^{2}=\left(\omega^{*}+\omega\right) \cdot \epsilon \cdot \eta=\xi^{n}=\alpha .
$$

Now for each irrational $z$, define

$$
\zeta_{z}=\sum_{r>z} \gamma(r)+\epsilon \cdot \eta+\sum_{r<z} \gamma(r) .
$$

By (17) and (18),

$$
\left(\omega^{*}+\omega\right) \cdot \zeta_{2} \text { is a solution of (i). }
$$

Consider irrationals $z$ and $z^{\prime}$ with $z<z^{\prime}$. Let $\sum_{Q} \phi(r)$ and $\sum_{Q} \phi^{\prime}(r)$ be scattered decompositions of $\zeta_{z}$ and $\zeta_{z^{\prime}}$. Choose a rational number $r_{0}$ such that $z<r_{0}<z^{\prime}$. The decomposition $\sum_{Q} \phi(r)$ has the property that there exists a smallest rational, $r^{\prime}$, for which $\phi\left(r^{\prime}\right)=\gamma\left(r_{0}\right)$, while $\sum_{Q} \phi^{\prime}(r)$ does not have the corresponding property. Hence, $\zeta_{z} \neq \zeta_{z^{\prime}}$, whence, by 3.13 ,

$$
\left(\omega^{*}+\omega\right) \cdot \zeta_{z} \neq\left(\omega^{*}+\omega\right) \cdot \zeta_{z^{\prime}}
$$

we now conclude from (19) that (i) has continuously many solutions. Moreover, it can be shown that all solutions are of the form (17).

We note that, in all the examples used in the above theorem, $\xi^{2}=\zeta^{2}$ implies $\xi^{n}=\zeta^{n}$ for every natural number $n$ greater than 2. A relevant open question is whether there exist types $\xi$ and $\zeta$ such that $\xi^{m}=\zeta^{m}$ and $\xi^{n} \neq \zeta^{n}$, where $m$ and $n$ are distinct inte, ers greater than 1 . Another problem which arises here is that of the existence of nondenumerable types having the same square. The problem will not be considered in this paper. However, as 
Tarski has pointed out, the answer is positive if one assumes the continuum hypothesis. For, $\left(\omega_{1} \cdot \eta_{1}\right)^{2}=\left(\omega_{1} \cdot \eta_{1}+\omega_{1}\right)^{2}=\omega_{1} \cdot \eta_{1}$, where $\omega_{1}$ is the smallest nondenumerable ordinal and $\eta_{1}$ is the "homogeneous normal type" of power $\boldsymbol{\aleph}_{1}$ defined in Hausdorff $\left[10\right.$, p. 181]. As is known, the existence of $\eta_{1}$ is equivalent to the continuum hypothesis.

\section{BIBLIOGRAPHY}

1. Anne C. Davis, Cancellation theorems for products of order types I, Bull. Amer. Math. Soc. Abstract 58-1-77.

2. - On order types whose squares are equal, Bull. Amer. Math. Soc. Abstract 58-3-276.

3. - Cancellation theorems for products of order types II, Bull. Amer. Math. Soc. Abstract 58-4-330.

4. - Surl'équation $\xi^{n}=\alpha$ pour des types d'ordre, C. R. Acad. Sci. Paris vol. 235 (1952) pp. 924-926.

5. - Square roots of some denumerable order types, Bull. Amer. Math. Soc. Abstract 59-1-110.

6. Anne C. Davis and W. Sierpiaski, Sur les types d'ordre distincts dont les carrés sont égaux, C. R. Acad. Sci. Paris vol. 226 (1952) pp. 850-852.

7. R. Fraĩssé, Sur la comparaison des types d'ordre, C. R. Acad. Sci. Paris vol. 226 (1948) pp. $1330-1331$.

8. S. Ginsburg, Some remarks on order types and decompositions of sets, Trans. Amer. Math. Soc. vol. 74 (1953) pp. 514-535.

9. - Fixed points of products and ordered sums of simply ordered sets, Proc. Amer. Math. Soc. vol. 5 (1954) pp. 554-565.

10. F. Hausdorff, Grundzïge der Mengenlehre, Leipzig, Viet, 1914 (Reprinted, New York, Chelsea, 1949.)

11. A. Lindenbaum and A. Tarski, Communication sur les recherches de la théorie des ensembles, Comptes Rendus des Séances de la Société des Sciences et des Lettres de Varsovie, vol. 19 (1926) Classe 3, pp. 299-330.

12. W. Sierpinski, Leçons sur les nombres transfinis, Paris, Gauthier-Villars, 1928.

13. - Sur la division des types ordinaux, Fund. Math. vol. 35 (1948) pp. 1-12.

14. Th. Skolem, Logisch-Kombinatorische Untersuchungen über die Erfüllbarkeit oder Beweisbarkeit mathematischer Sätze nebst einem Theoreme über dichte Mengen, Skrifter utget av Videnskapsselskapet i Kristiania, I klasse, 1920, no. 4.

15. A. Tarski, Ordinal algebras, Amsterdam, North Holland Publishing Company, 1956.

University of California, Davis, Calif. 